\title{
FORMATION OF GIANT LUMINOUS ARCS AND ARCLETS USING AN OPTICAL GRAVITATIONAL LENS EXPERIMENT
}

\section{J. SURDEJ}

STScI, 3700 San Martin Drive, Baltimore, MD 21218, USA, Member of the Astrophysics Division, Space Science Department of the European Space Agency, and Directeur de Recherches au FNRS, Belgium

S. REFSDAL

Hamburg Observatory, Gojenbergsweg 112, D-21029 Hamburg, Germany

\section{AND}

\section{A. POSPIESZALSKA-SURDEJ \\ STScI, 3700 San Martin Drive, Baltimore, MD 21218, USA}

\begin{abstract}
It is well known that simple optical lenses simulating light deflection due to a foreground object may easily reproduce all types of image configurations observed among known multiply imaged QSOs and AGN (cf. simulations in Refsdal and Surdej 1992, 1994, Surdej et al. 1993). Such an optical lens experiment can also be used to simulate the formation of multiple giant luminous arcs and arclets seen near massive foreground galaxy clusters. The optical setup used to make this experiment is shown in Fig. 1 (a similar setup was presented during the Melbourne conference). A compact light source is located on the right side (not very clearly seen), then comes to the left a 'spiral galaxy disk' optical lens (manufactured by the authors) and a cardboard perforated by multiple pinholes. In the absence of light deflection (i.e. by simply removing the optical lens), direct (non distorted) images of the background galaxies are seen by an observer, like those projected on the distant white screen (see Fig. 2a). When the optical lens is inserted between the light source and the multiple pinholes screen (cf. Fig. 1), images of the background galaxies get distorted (arclets) and/or transformed into multiple images, including giant luminous arcs (see Figs. $2 \mathrm{bc}$ for examples). We showed during the conference that by covering the pinholes with various transparent colored filters and translating the multi-
\end{abstract}




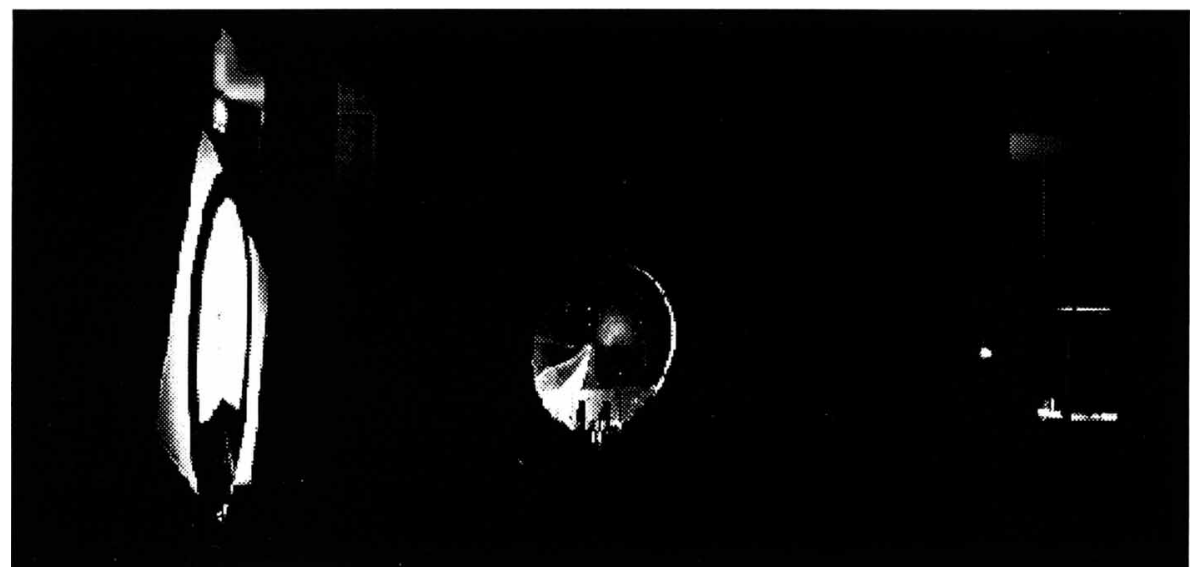

Figure 1. Setup of the optical gravitational lens experiment used to simulate distorted images (cf. Figs. 2bc) of background galaxies (Fig. 2a) by a foreground cluster (see text)
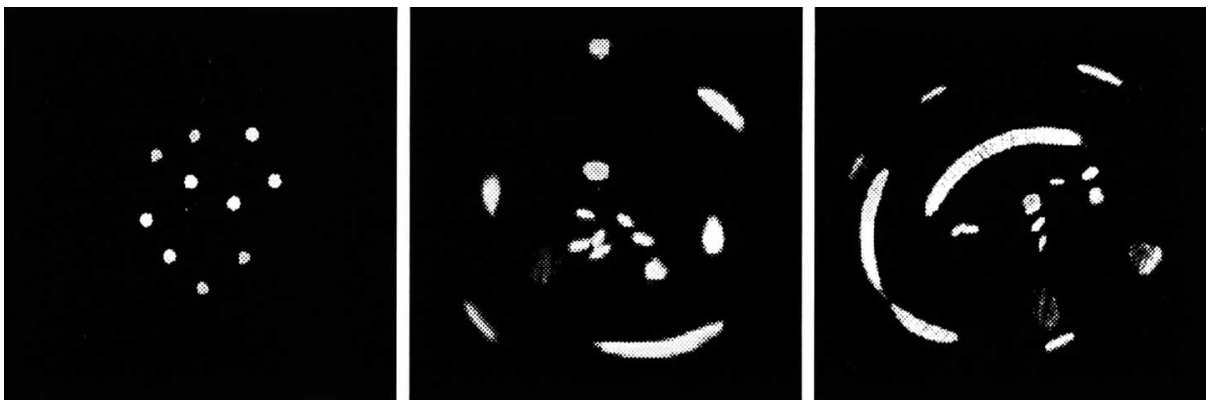

Figure 2. Giant luminous arcs and arclets (Figs. 2bc) resulting from the gravitational lens distortion of background galaxies (Fig. 2a) by a foreground cluster

ple pinholes screen, it was possible to reproduce, in a very inexpensive way, distorted images of background galaxies like the very impressive strong lensing artifacts seen behind the rich cluster Abell 1689 ( $\mathrm{cf}$. the color videotape of simulations by Tony Tyson).

\section{References}

Refsdal, S., \& Surdej, J., 1992, in Highlights of Astronomy, ed. J. Bergeron, 9, 3

Refsdal, S., \& Surdej, J., 1994, Rep Prog Phys, 56, 117

Surdej, J., Refsdal, S., \& Pospieszalska-Surdej, A., 1993, in Gravitational Lenses in the Universe, eds. J. Surdej, D. Fraipont-Caro, E. Gosset, S. Refsdal \& M. Remy, (Liège: Université de Liège) 199 